\title{
CATETER CENTRAL DE INSERÇÃO PERIFÉRICA: PERCEPÇÕES DA EQUIPE DE ENFERMAGEM
}

\author{
Janislei Giseli D. Stocco ${ }^{1}$, Karla Crozeta ${ }^{2}$, Liliana Maria Labronici³ ${ }^{3}$, Mariluci Alves Maftum ${ }^{4}$, Marineli Joaquim Meier ${ }^{5}$
}

RESUMO: Pesquisa descritiva e exploratória realizada na Unidade de Terapia Intensiva Pediátrica de um hospital de Curitiba em setembro de 2008. Teve por objetivos refletir sobre o papel do profissional de enfermagem no cuidado ao paciente com cateter venoso central de inserção periférica (PICC) e discutir sobre o cuidado do paciente com PICC sustentado na visão tecnológica. Participaram do estudo uma enfermeira, cinco técnicos e quatro auxiliares de enfermagem. Os dados foram coletados mediante a técnica de discussão durante quatro encontros e organizados nas categorias: Aspectos do cuidado de enfermagem ao paciente com PICC e Visão tecnológica no cuidado ao paciente com PICC. Concluiu-se que a percepção sobre tecnologia está associada a máquinas e equipamentos, mas a equipe reconheceu a tecnologia como impactante na qualidade de vida do paciente; e reconheceu também a necessidade de socialização do conhecimento, mediante estratégias de educação permanente.

PALAVRAS-CHAVE: Cuidados de enfermagem; Cateterismo venoso central; Tecnologia.

\section{PERIPHERAL INSERTION CENTRAL CATHETER: PERCEPTIONS OF THE NURSING TEAM}

\begin{abstract}
Descriptive, exploratory research carried out in a Paediatric Intensive Therapy Unit in Curitiba in September 2008. The aims were to reflect on the role of the nursing professional in the care of the patient with a peripherally inserted central catheter (PICC) and to discuss from the technological point of view the care of patients with PICCs. The participants were a nurse, five technicians and four nursing auxiliaries. The data were collected through discussion technique. during four meetings and organized into two categories: aspects of nursing care of the patient with a PICC and the technical viewpoint in the care of the patient with a PICC. It was concluded that perception of technology is associated with machines and equipment, but that the team recognised both the technology's impact on the patient's quality of life, and the need to socialize the knowledge, through lasting educative strategies.
\end{abstract}

KEYWORDS: Nursing Care; Central Venous Catheterization; Technology.

\section{CATÉTER CENTRAL DE INSERCIÓN PERIFÉRICA: PERCEPCIONES DEL EQUIPO DE ENFERMERÍA}

RESUMEN: Investigación descriptiva y exploratoria realizada en la Unidad de Terapia Intensiva Pediátrica de un hospital de Curitiba, en septiembre de 2008. Tuvo por objetivos reflexionar acerca del papel del profesional de enfermería en el cuidado al paciente con catéter venoso central de inserción periférica (PICC) y discutir sobre el cuidado del paciente con PICC sustentado en la perspectiva tecnológica. Participaron del estudio una enfermera, cinco técnicos y cuatro auxiliares de enfermería. Los datos fueron recogidos por la técnica de discusión durante cuatro encuentros y organizados en las categorías: Aspectos del cuidado de enfermería al paciente con PICC y Visión tecnológica en el cuidado al paciente con PICC. Se concluyó que la percepción sobre tecnología está asociada a máquinas y aparatos, pero el equipo reconoció la tecnología como impactante en la cualidade de vida del paciente; y reconoció también la necesidad de socialización del conocimiento, por medio de estrategias de educación permanente.

PALABRAS CLAVE: Cuidados de enfermería; Cateterismo venoso central; Tecnología.

${ }^{1}$ Enfermeira. Mestre em Enfermagem. Membro do Grupo de Pesquisa Tecnologia e Inovação em Saúde: Fundamentos para a Prática Profissional da Universidade Federal do Paraná - TIS UFPR.

${ }^{2}$ Enfermeira. Mestre em Enfermagem. Membro do TIS UFPR.

${ }^{3}$ Enfermeira. Professora em Enfermagem do Departamento de Enfermagem e do Programa de Pós-Graduação em Enfermagem da Universidade Federal do Paraná - PPGENF UFPR. Líder do Grupo de Estudos Multiprofissional em Saúde do Adulto da UFPR - GEMSA. ${ }^{4}$ Enfermeira. Doutora em Enfermagem. Professora do Departamento de Enfermagem e do PPGENF UFPR. Vice-líder do NEPECHE. ${ }^{5}$ Enfermeira. Doutora em Enfermagem. Professora do Departamento de Enfermagem e do PPGENG UFPR. Líder do TIS UFPR.

Autor correspondente:

Mariluci Alves Maftum

Universidade Federal do Paraná

R. Clemente Tesseroli, 90 - 81520-190 - Curitiba-PR-Brasil

Recebido: 02/09/10

E-mail:maftum@ufpr.br

Aprovado: 04/02/11 


\section{INTRODUÇÃO}

O avanço tecnológico na área da saúde e, em especial, na terapia intravenosa, propicia o uso de materiais como o cateter venoso central de inserção periférica (PICC). Este requer dos profissionais de enfermagem a apropriação de conhecimento específico para sua utilização. Entretanto, por vezes, percebe-se que o uso de recursos tecnológicos tende à valorização dos procedimentos técnicos (fazer) em detrimento do saber (conhecimento).

Na área da saúde, a tecnologia pode ser compreendida sob duas perspectivas: materiais/equipamentos, e conhecimentos, uma vez que é um instrumento essencial na construção do "saber-fazer", permeado e influenciado por bases teórico-práticas ${ }^{(1)}$. Para tanto, as tecnologias na saúde foram agrupadas em três categorias: dura, leve-dura e leve. A primeira é representada pelos materiais concretos, tais como equipamentos, mobiliários permanentes ou material de consumo; a leve-dura inclui saberes estruturados e representados pelas disciplinas da área da saúde; e a leve se expressa nos processos de produção da comunicação, das relações e de vínculos que conduzem ao encontro do usuário com ações de saúde ${ }^{(1)}$.

O conceito de Tecnologia na Enfermagem assume significado peculiar:

compreende o conhecimento humano (científico e empírico) sistematizado, requer a presença humana, visa à qualidade de vida e se concretiza no ato de cuidar, considerando a questão ética e o processo reflexivo $^{(2: 167)}$.

Esse conceito extrapola o uso de materiais e equipamentos, visto que engloba diversos saberes que conduzem à finalidade proposta pelo processo produtivo do profissional enfermeiro, o cuidado ${ }^{(2)}$.

A Tecnologia em Enfermagem se efetiva na relação entre o saber-fazer, mediante um processo reflexivo que considera a interação entre enfermeiro e cliente. A interrelação entre saber e fazer caracteriza a visão tecnológica na Enfermagem ${ }^{(3)}$, perspectiva adotada neste estudo. Destarte, o PICC é compreendido como uma tecnologia, pois representa a extensão do paciente como parte integrante do processo de cuidado, requer a presença humana e se efetiva no ato de cuidar, bem como exige a relação entre saber e fazer para o melhor cuidar.

Assim, adotar essa visão tecnológica ${ }^{(2)}$ propicia uma compreensão ampliada acerca do uso do PICC, uma vez que enfatiza aspectos como motivação, reconhecimento, contribuição para o alcance dos objetivos do processo de trabalho, qualidade e especificidade sobre os resultados esperados, e segurança e competência na realização do cuidado.

Isso contribui na tomada de decisão dos profissionais para a indicação do cateter em situações específicas, que incluem terapias de longa duração (acima de uma semana); administração de nutrição parenteral (com dextrose acima de 10\%), infusão de medicamentos vesicantes, irritantes ou vasoativos; soluções hiperosmolares ou com pH não-fisiológico (alguns antibióticos e quimioterápicos antineoplásicos); administração de hemoderivados, verificação de pressão venosa central e coleta de sangue ${ }^{(4-5)}$.

Ressalta-se, ainda, a importância de capacitar a equipe de enfermagem para o manuseio do dispositivo, pois as perdas do cateter estão relacionadas, principalmente, aos cuidados referentes à sua manutenção. Assim, considera-se que a sensibilização, o envolvimento e a valorização da equipe de enfermagem, no que concerne ao cuidado do paciente com PICC, na visão tecnológica, contribuem para a reflexão sobre a prática e a diminuição das perdas do dispositivo.

Por se tratar de um equipamento de alto custo, sua utilização no cenário em que este estudo foi desenvolvido é restrita. Os benefícios à criança incluem diminuição de número de punções, acesso venoso central para infusão de drogas irritantes e/ou vesicantes, prevenção de flebites ou queimaduras por extravasamento, redução de dor decorrente de punções frequentes, diminuição do número de flebotomias ${ }^{(6)}$, entre outros que envolvem o conforto e bem-estar.

Ao se considerar que o cuidado do paciente com PICC compreende aspectos peculiares à manutenção desse dispositivo, a fim de assegurar sua permanência e a diminuição de complicações, percebeu-se a necessidade de desenvolver este estudo de cunho educativo, que teve como objetivos refletir sobre o papel do profissional de enfermagem no cuidado ao paciente com PICC e discutir sobre o cuidado do paciente sustentado na visão tecnológica.

\section{METODOLOGIA}

Esta pesquisa foi precedida da aprovação pelo Comitê de Ética em Pesquisa do Hospital de realização do estudo, CAAE 0234.0.208.000-08, por atender aos aspectos das Resoluções CNS 196/96 e demais Diretrizes e Normas Regulamentadoras de Pesquisa 
envolvendo seres humanos.

Trata-se de um estudo qualitativo, descritivo e exploratório da prática assistencial de enfermagem, desenvolvido de setembro a novembro de 2008, em uma Unidade de Terapia Intensiva Pediátrica (UTI Ped) de um Hospital Universitário de Curitiba, com uma equipe composta por 20 profissionais de enfermagem, dos quais 10 (uma enfermeira, cinco técnicos e quatro auxiliares de enfermagem) participaram da pesquisa.

Foram incluídos neste estudo os dados dos colaboradores que aceitaram participar e assinaram o Termo de Consentimento Livre e Esclarecido, independentemente do turno de trabalho, e que participaram de todos os encontros. O convite foi realizado verbalmente em todos os turnos de trabalho e por escrito, afixado no mural de recados da UTI Ped.

A técnica utilizada para a coleta de dados foi a Discussão de Grupo, que permite complementação de informações sobre conhecimentos específicos conforme suas crenças, percepções e atitudes. O momento de discussão proporciona o aparecimento de uma situação singular e específica do grupo $^{(7)}$, pois surgem opiniões, relevâncias e valores dos indivíduos. As informações que surgiram dos encontros foram registradas por gravação em fita magnética e anotações de campo.

Foram realizados quatro encontros, em dois horários (das $12 \mathrm{~h} 00$ às $13 \mathrm{~h} 00$ e das $18 \mathrm{~h} 00$ às $19 \mathrm{~h} 00$ ), na sala de aula da UTI Ped, a fim de facilitar a participação do maior número de colaboradores. Os encontros ocorreram nas quartas e quintas-feiras, conforme sugestão prévia dos participantes.

No primeiro encontro, solicitou-se aos participantes que respondessem individualmente, e por escrito, três questões: $\mathrm{O}$ que é cuidado para você? $\mathrm{O}$ que é tecnologia? Qual o papel que você exerce no cuidado ao paciente com o PICC? Estas questões serviram para subsidiar as discussões dos encontros subsequentes.

No segundo encontro, foram debatidas as questões respondidas anteriormente e discutidas à luz da visão tecnológica, com o auxílio de um texto específico sobre o assunto, e do conceito de tecnologia de Meier ${ }^{(2)}$, o que propiciou aos participantes expressarem sua opinião sobre esse conceito e sua relação com o cuidado do paciente com o PICC.

$\mathrm{O}$ tema para o terceiro encontro foi "O cuidado do paciente com o PICC sustentado na visão tecnológica". Como recursos, foram utilizados um vídeo específico sobre os aspectos relacionados ao cuidado e manutenção do dispositivo e o protocolo que a unidade possui para inserção do cateter. Este protocolo compreende aspectos específicos sobre o paciente (idade, patologia, exames complementares, aspectos da rede venosa e pele), o cateter (lote, french, comprimento, tentativas de inserção), os curativos (data, material utilizado, fixação do dispositivo, aspectos da pele, sinais flogísticos) e a retirada (motivo, dificuldades, comprimento retirado).

No último encontro, foi solicitado aos participantes que fizessem sugestões para solucionar os problemas identificados no cuidado ao paciente com PICC.

Os dados foram analisados segundo a Análise Temática de $\operatorname{Bardin}^{(8)}$, que se configura em três etapas: pré-análise, exploração do material e tratamento dos resultados. A pré-análise ocorreu por meio da transcrição das falas, seguida da leitura flutuante. A exploração do material constituiu-se da compilação, enumeração e rejeição dos dados, e os sujeitos foram codificados pela letra P (Participante). Por fim, ocorreu a organização dos resultados em categorias temáticas a partir dos relatos dos participantes e que foram interpretadas com base no referencial da Tecnologia em Enfermagem ${ }^{(2)}$.

\section{RESULTADOS}

As categorias que emergiram a partir da análise dos dados foram: Aspectos do cuidado de enfermagem ao paciente com PICC, e A visão tecnológica no cuidado ao paciente com PICC.

Com relação aos Aspectos do cuidado de enfermagem a paciente com PICC, os participantes evidenciaram aspectos específicos, tais como indicações e contraindicações, manutenção, complicações e retirada do cateter, o que demonstrou que possuíam conhecimentos básicos acerca do PICC, porém apontaram dúvidas quanto à indicação do dispositivo:

\section{Podemos usar em todas as crianças? (P2)}

Lavar o cateter com soro fisiológico após cada medicação e administrar solução de heparina a cada seis horas para evitar obstrução; observar fluxo e refluxo de sangue; cuidar para não tracionar o cateter. (P6)

Observar permeabilidade; cuidar com assepsia durante o manuseio; cuidados com manutenção do cateter. (P7) 
Observar fluxo e refluxo de sangue; lavar com soro fisiológico; cuidado para não tracionar o cateter. (P5)

É um procedimento altamente invasivo, por isso se deve cuidar com assepsia durante a administração de medicamentos e durante o manuseio. (P3)

Só deve ser utilizadas seringas acima de $5 \mathrm{ml}$, pois abaixo deste valor rompe o cateter. (P1)

Observa-se que os participantes reconhecem a necessidade de cuidados específicos com o PICC, e que a permanência do mesmo e possíveis complicações estão relacionadas à manutenção adequada do dispositivo.

Quanto à categoria sobre a visão tecnológica no cuidado ao paciente com PICC, os participantes deram ênfase à importância da tecnologia na sua prática cotidiana, sendo que o predomínio das percepções evidenciou uma visão relacionada a máquinas e equipamentos:

A tecnologia são equipamentos e máquinas, entre outros, que foram inventados para nos ajudar, como o PICC. (P1)

Tecnologia é o equipamento para facilitar nosso trabalho. (P2)

A tecnologia é um conjunto de conhecimentos que, de maneira organizada, se transforma em equipamentos, procedimentos com determinada finalidade. (P3)

São os materiais criados para auxiliar nosso trabalho. É o termo que envolve conhecimentos técnicos, cientificos; são materiais criados e utilizados a partir de tal conhecimento. (P5)

Após a teorização das discussões de grupo, os participantes externaram alguns aspectos relacionados ao cateter, o qual, por si, não satisfaz a necessidade do paciente, pois a efetividade do cuidado requer o relacionamento interpessoal, e a necessidade dos profissionais de refletir sobre suas ações. Os participantes externaram, ainda, que o uso da tecnologia implica em ponderar também sobre a qualidade de vida do paciente em relação ao uso racional dos aparatos tecnológicos:

O PICC precisa da presença humana para ser cui- dado. (P8)

Sem a pessoa presente, ele não funciona; precisa de alguém que cuide deste cateter. (P3)

Percebi que preciso refletir sobre o que faço. (P4)

Tenho que pensar na qualidade de vida do paciente para usar a tecnologia. (P9)

A partir das discussões, os participantes apontaram sugestões para a resolução dos problemas por eles detectados como, por exemplo, a construção de um documento para consulta de todos os profissionais envolvidos no cuidado ao paciente com PICC, bem como a necessidade de educação permanente com a equipe de enfermagem:

\section{A construção do POP. (P3)}

Realizar educação continuada com os outros turnos. (P2)

Se conseguir um boneco e o PICC para demonstrar os cuidados; instigar a pessoa para ver se ela aprendeu a cuidar na visão tecnológica. (P9)

Deixar no box os cuidados por escrito, para não esquecer e, em caso de dúvidas, poder saná-las. (P8)

Os participantes percebem a importância do envolvimento de toda a equipe de enfermagem para cuidado do paciente com PICC na visão tecnológica, para tanto propõem a educação continuada e a extensão destas discussões para os demais colegas.

\section{DISCUSSÃO}

Os participantes citaram uma das questões centrais acerca da discussão sobre o conceito tecnologia: a restrição do conceito a máquinas e equipamentos. No cuidado de enfermagem, a Tecnologia envolve saberes e habilidades que precisam ser distinguidos de aparelhos tecnológicos e, portanto, não se restringe ao tangível, mas ao resultado de um trabalho que envolve ações que apresentam uma finalidade ${ }^{(1)}$. Além disso, considera-se a questão ética e o processo reflexivo ${ }^{(2)}$.

O cuidado na perspectiva tecnológica sugere repensar a capacidade do ser humano em inovar e transformar seu cotidiano, com vistas à melhoria da 
qualidade de vida e à satisfação pessoal. A tecnologia se efetiva no ato de cuidar, uma vez que o cuidado requer a utilização adequada desta, que a caracteriza como elemento mediador da racionalidade e da subjetividade, da intuição e da emoção, utilizando-se a sensibilidade para fortalecer e qualificar o cuidado de enfermagem ${ }^{(9)}$.

Após a teorização sobre tecnologia, esta foi mencionada pelos participantes como algo que, permeada pelo conhecimento técnico, científico e humano organizado, tem por finalidade trazer melhoria no processo de cuidar.

$\mathrm{Na}$ Enfermagem, a tecnologia é um elemento que viabiliza a relação entre profissional e o paciente, resultando em um cuidado único, essencial para construção do saber-fazer. Na relação entre cuidado e tecnologia, interligam-se conhecimentos e instrumentos que fundamentam e delimitam modos de implementação, modos sistematizados de saber-fazer o cuidar humano. A tecnologia permeia o processo de trabalho em saúde, contribui com a construção do conhecimento $^{(9)}$, e integra o saber com o fazer mediante o processo reflexivo que considera a interação profissional e pessoal ${ }^{(3)}$. Portanto, a ligação entre tecnologia e cuidado implica em conhecimento científico, utilizado de maneira crítico-reflexiva, visto que é um importante componente na prática de cuidar.

$\mathrm{Na}$ visão tecnológica, os participantes mencionaram alguns aspectos relacionados ao cateter, evidenciando que, sozinho, o dispositivo não atende às necessidades do paciente. $\mathrm{O}$ emprego da tecnologia é envolto por questões éticas, uma vez que cabe ao profissional a decisão sobre o uso que fará dela, e ele, mediante postura crítica, pode aprová-la ou reprová$1 \mathrm{a}^{(10)}$, de acordo com as necessidades do paciente e com vistas à qualidade de vida.

Dessa forma, perceber a tecnologia de maneira abrangente propicia o uso consciente do PICC, pois sua utilização requer cuidados, intervenções nos problemas sociais, uso de métodos e outras práticas que fazem parte do processo de trabalho do enfermeiro ${ }^{(1)}$.

Assim, constatou-se que a manutenção do cateter, o tempo de permanência, a indicação do dispositivo e a administração de heparina ou solução salina isotônica foram referidos pelos participantes como cuidados essenciais ao paciente com PICC. Isso evidencia o caráter tecnicista, preocupado apenas na execução do cuidado técnico. Disso emerge a necessidade de discussão do cuidado ao paciente com PICC abordado com outros elementos estéticos, éticos, sociais e políticos, a fim de contemplar a visão tecnológica.

Dentre os cuidados técnicos, a literatura aponta que a infusão de hemoderivados e hemocomponentes, bem como a coleta de sangue, devem ocorrer acima de 3,8 french, a fim de evitar a sua obstrução ${ }^{(4,11)}$. Quanto às seringas, deve-se usar somente as acima de $5 \mathrm{ml}$, pois, quanto menor a seringa, maior a pressão, o que pode causar ruptura do cateter. A técnica asséptica durante a inserção e manutenção, bem como a observação das orientações do Infusion Nurse Society (INS) e dos Centers for Diseases Control and Prevention (CDC) com relação às trocas de equipo e dispositivos, tempo de permanência do cateter e tempo de duração, troca e validade de infusão, previnem infecções ${ }^{(12)}$.

Destaca-se que o método de salinização-administração do medicamento-salinização e heparinização (SASH) é aconselhado e previne a ocorrência de incompatibilidade entre as soluções infundidas, além de manter o bom funcionamento do cateter. $\mathrm{O}$ volume de solução salina varia de 10 a $20 \mathrm{ml}$ após a infusão de soluções hiperosmolares, medicamentos vesicantes e irritantes, hemoderivados ou coleta de amostra sanguínea, tamanho do cateter e idade da criança ${ }^{(12)}$.

Os volumes infundidos, bem como a quantidade de heparina a ser administrada, devem ser padronizados nos protocolos dos serviços, considerando-se os fatores relacionados ao paciente (idade, peso, fisiopatologia) ${ }^{(5)}$.

Ressalta-se que vasos delgados, de difícil identificação, comprometidos por edema, eritema e hematomas (causados por repetidas punções venosas ou cirurgias traumáticas), constituem os maiores problemas para inserção e manutenção do cateter. A indicação deve ser precoce, sendo a primeira escolha ${ }^{(13)}$. Dessa forma, o dispositivo não é recomendado para todos os pacientes; cabe ao enfermeiro, juntamente com a equipe médica, avaliar e indicar ou não a inserção do PICC.

Os participantes reconheceram a necessidade de educação permanente, o que caracteriza um momento oportuno de discussão sobre o cuidado de enfermagem ao paciente com cateter PICC. Devido à especificidade do cateter, destaca-se que a educação permanente nos serviços de saúde é capaz de suprir lacunas, fazer parte do processo de trabalho e proporcionar reflexão e atualização das práticas no cotidiano do trabalhador ${ }^{(14)}$.

O artigo 17 da Resolução no 7, da Agência Nacional de Vigilância Sanitária, dispõe que a equipe da UTI deve participar de um programa de educação continuada, contemplando, no mínimo: normas e rotinas técnicas desenvolvidas na unidade; incorporação de 
novas tecnologias; gerenciamento dos riscos inerentes às atividades desenvolvidas na unidade e segurança de pacientes e profissionais; e prevenção e controle de infecções relacionadas à assistência à saúde ${ }^{(15)}$.

Ressalta-se que a Portaria GM/MS no 198/2004 institui a Política Nacional de Educação Permanente em Saúde, como estratégia do Sistema Único de Saúde, para a formação e o desenvolvimento dos profissionais, e busca agregar o aperfeiçoamento individual e institucional ${ }^{(16)}$. Assim, o interesse do grupo pelo processo educativo vem ao encontro dessa estratégia, bem como atende os pressupostos do referencial de Tecnologia em Enfermagem, uma vez que enfatiza a reflexão e atualização da equipe de enfermagem.

\section{CONSIDERAÇÕES FINAIS}

O uso do PICC no local em que o estudo foi realizado ainda é restrito. Assim, o presente estudo evidenciou a necessidade da equipe de enfermagem refletir sobre o cuidado ao paciente que utiliza esse dispositivo.

Nas discussões de grupo, ficou evidente a concepção de tecnologia restrita a máquinas e equipamentos e a ênfase nos cuidados técnicos aos pacientes com cateter PICC. Ressalta-se que o referencial de Tecnologia em Enfermagem não era do conhecimento dos participantes. Assim, considera-se que esse favoreceu a sensibilização e reflexão dos profissionais sobre a prática de cuidado do paciente com PICC, por fomentar dúvidas e fazer emergir a necessidade de ampliação do conhecimento da equipe em relação ao cateter.

Essa concepção teórica ainda é desconhecida por muitos profissionais de saúde. Deste modo, o desenvolvimento de estudos que utilizem o referencial é imperioso para a apropriação da visão tecnológica na prática da enfermagem, a fim de transpor a percepção da tecnologia como restrita às máquinas e aos equipamentos, e valorizar o conhecimento e relacionamento interpessoal no cuidado.

Durante as discussões grupais, houve a preocupação em socializar os conhecimentos relativos ao PICC e o referencial de Tecnologia em Enfermagem com os demais profissionais que realizam o cuidado do paciente com o cateter.

Ressaltou-se a necessidade de desenvolvimento de um processo de educação permanente, o qual sendo operacionalizado, poderá contribuir para a qualificação da equipe para o cuidado.

\section{REFERÊNCIAS}

1. Merhy EE, Onocko R, organizadores. Praxis en la salud: un desafio para lo público. Buenos Aires: Lugar Editorial; 1997.

2. Meier MJ. Tecnologia em enfermagem: desenvolvimento de um conceito [tese]. Florianópolis (SC): Universidade Federal de Santa Catarina; 2004.

3. Nascimento SR, Meier MJ. Uma visão tecnológica do exame físico. In: Carraro TE, Westephalen MEA. Metodologias para a assistência de enfermagem: teorizações, modelos e subsídios para a prática. Goiânia: AB; 2001. p. 127-35.

4. Vendramin P. Cateter central de inserção periférica (CCIP). In: Harda MJCS, Rego RC, organizadores. Manual de terapia intravenosa em pediatria. São Paulo: ELLU; 2005. p. 75-95.

5. Secoli SR, Kishi HM, Carrara D. Inserção e manutenção do PICC: aspectos da prática clínica de enfermagem em oncologia. Prática Hospitalar. 2006;7(47):155-62.

6. Vendramin P, Pedreira MLG, Perterlini MAS. Cateteres centrais de inserção periférica em crianças de hospitais do município de São Paulo. Rev Gaúch Enferm. 2007;28(3):331-9.

7. Minayo MCS. O desafio do conhecimento: pesquisa qualitativa em saúde. São Paulo: Hucitec; 2004.

8. Bardin L. Análise de conteúdo. Lisboa: Edições 70; 2000.

9. Rocha PK, Prado ML, Wal ML, Carraro TL. Cuidado e tecnologia: aproximação através do cuidado. Rev Bras Enferm. 2008;61(1):113-6.

10. Crozeta K, Stocco JGD, Labronici LM, Meier MJ. Interface entre a ética e um conceito de tecnologia em enfermagem. Acta Paul Enferm. 2010;23(2):239-43.

11. Jesus VC, Secoli SR. Complicações acerca do cateter venoso central de inserção periférica (PICC). Cienc Cuid Saúde. 2007;6(2):252-60.

12. Gorski LA, Czaplewski LM. Peripherally inserted central catheteres. J Infus Nurs. 2004;27(6):399-409.

13. Camara D. Minimizing risks associated with peripherally inserted central catheter in the NICU. MCN Am J Matern Child Nursing. 2001;26(1):17-21. 
14. Lino MM, Backes VMS, Schmidt SMS, Ferraz F, Prado ML, Martins T. A realidade da educação continuada na enfermagem nos serviços públicos de saúde de Florianópolis. Online Braz J Nurs. [Internet] 2007;6(0) [acesso em 6 nov 2008]. Disponível: http:// www.objnursing.uff.br/index.php/nursing/article/ view/619/147.

15. Ministério da Saúde (BR). Agência Nacional de Vigilância Sanitária. Resolução n. 7, de 24 de fevereiro de 2010. Dispõe sobre os requisitos mínimos para funcionamento de unidades de terapia intensiva e dá outras providências. Diário Oficial da União [Internet] 25 fev 2010 [acesso em 20 ago 2010]. Disponível: http:// amib.org.br/pdf/rdc-07-2010.pdf.

16. Ministério da Saúde (BR). Portaria GM/MS n. 198/04, de 13 de fevereiro de 2004. Institui a política Nacional de educação permanente em saúde para formação e o desenvolvimento de trabalhadores para o setor dá outras providências. Diário Oficial da República Federativa do Brasil, Brasília, 14 fev. 2004. Seção 1:1. 